\title{
Comet 103P/Hartley 2 at perihelion: gas and dust activity ${ }^{\star}$
}

\author{
L. M. Lara ${ }^{1}$, Z.-Y. Lin ${ }^{1}$, and K. Meech ${ }^{2}$ \\ 1 Instituto de Astrofísica de Andalucía (CSIC), Glorieta de la Astronomía s/n, 18008 Granada, Spain \\ e-mail: [lara;zlin]@iaa.csic.es \\ 2 Institute for Astronomy, 2680 Woodlawn Drive, Honolulu HI 96822, USA \\ e-mail: meech@ifa.hawaii.edu
}

Received 15 March 2011 / Accepted 7 June 2011

\begin{abstract}
Context. The comet 103P/Hartley 2, target of the EPOXI mission (NASA), was supposed to be observed for 3 days around its perihelion, from October 27 to 29, 2010, but photometric data were obtained only on October 27 and 29, 2010. On both dates, the comet visibility was not optimal due to its proximity to the Moon, as projected on the plane of the sky, whereas on October 28 , the comet could not be observed at all.

Aims. The goal of the campaign was to give ground support to the EPOXI mission by establishing a baseline of activity at perihelion to be compared with in situ activity observed by the space mission about 7 days later on Nov. 4, 2010. We aimed to assess gas and dust production rates, to study the gas and dust coma morphology, to investigate the behaviour of the refractory component by analysing the dust colour variations with date and with projected cometocentric distance, $\rho$, and to determine the slope of the surface brightness profiles, B, as a function of $\rho$.

Methods. Long-slit spectra and optical broad- and narrowband images were acquired with the instrument ACAM mounted on the William Herschel Telescope (WHT) at La Palma Observatory. We investigated the evolution of the dust coma morphology from the images acquired with specific continuum cometary filters (in the blue and red wavelength region) with image-enhancing techniques. We studied (1) the gas and dust production rates; (2) the dust radial brightness profiles; (3) the profiles of the $\mathrm{CN}_{2} \mathrm{C}_{2}, \mathrm{C}_{3}$ and $\mathrm{NH}_{2}$ column densities, and (4) the $\mathrm{CN}$ and $\mathrm{C}_{3}$ coma morphologies. The dust and gas profiles were azimuthally averaged, as well as measured in both the E-W direction ( $\sim$ Sun-antisolar direction) and in a direction defined by the slit orientation at PA 70 to 250 degrees.

Results. The morphological analysis of the dust coma reveals only one structure. Aside from the dust tail in the west direction, a bright jet is detected in images acquired on October 27 at 03:00-04:00 UT. This jet turns on and off and it is not clearly detected at any time on the images obtained during October 29. This structure is enhanced by making use of the radial renormalization and the LarsonSekanina method. It is also confirmed by the distortion of the isophotes at the same position angle (PA). The Afo parameter, a proxy to the dust production rate, and the gas $\left(\mathrm{CN}, \mathrm{C}_{3}, \mathrm{C}_{2}\right.$, and $\left.\mathrm{NH}_{2}\right)$ production rate, $Q_{i}$, have been measured at perihelion, $r_{\mathrm{h}} \approx 1.058 \mathrm{AU}$. The quotient $Q_{\mathrm{C}_{2}} / Q_{\mathrm{CN}} \sim 1.3$ places $103 \mathrm{P} /$ Hartley 2 as a typical comet in terms of long-chain hydrocarbon abundance. The gas-to-dust mass ratio is $\sim 3-6$, indicating that 103P/Hartley 2 is a relatively gas-rich comet. At perihelion, Af $\rho$, as measured in a circular aperture of $\sim 4700 \mathrm{~km}$ ranges from $\sim 60 \mathrm{~cm}$ in the blue to $\sim 110 \mathrm{~cm}$ in the red, which indicates an overall change in the optical properties of the dust grains. On the other hand, the $A f \rho$ is rather stable in the innermost coma when it is computed from the spectroscopic measurements within several continuum spectral ranges from 482-685 nm. Both 2D dust colour maps and profiles in the directions imposed by the slit indicate that there are variations with $\rho$ with a trend towards bluer dust colour with increasing $\rho$. This could indicate sublimation of ices as the cameras on board the EPOXI mission have shown. The average dust reddening is $\sim 24 \% / 100 \mathrm{~nm}$. The azimuthally averaged surface brightness profiles of the continuum from the broad band images can be well fitted with $m \sim 1$ in the tail direction, whereas in the opposite direction the dust profiles are much flatter at $\rho \leq 10000 \mathrm{~km}$. The azimuthally averaged profiles of the comet images acquired with the blue and red continuum cometary filters show a nominal behaviour of $\log B \sim-m \log \rho$ with $m \sim 1$.
\end{abstract}

Key words. comets: general - comets: individual: 103P/Hartley 2

\section{Introduction}

The most important scientific rationale for studying comets is to retrieve information on their origin, on their relationship with interstellar, and on interplanetary material and implications for the formation of the Solar System. Deep Impact was the first mission to look beneath the surface of a comet (i.e. 9P/Tempel 1) (A'Hearn et al. 2005). The mission has provided us with unique information about primordial material in comets, global nucleus properties (density, material strength, ageing of cometary

* Based on observations made with the William Herschel Telescope (WHT) operated on the island of La Palma by the Isaac Newton Group in the Spanish Observatorio del Roque de los Muchachos of the Instituto de Astrofísica de Canarias. surface), and basic properties such as the crater formation on celestial bodies (A'Hearn 2007; Thomas et al. 2007).

Following the Deep Impact encounter with 9P/Tempel 1, the spacecraft was left on an orbit that gave the possibility of redirecting it to $103 \mathrm{P} /$ Hartley 2 . The comet nucleus was planned to be flown by on November 4, 2010. Comet 103P/Hartley 2 was discovered in 1984 (Hartley 1984). It is an ecliptic comet that moved in the vicinity of the 1:1 mean motion resonance with Jupiter until 1875. After that date, a series of encounters with Jupiter reduced its perihelion from $2.9 \mathrm{AU}$ to $\sim 1 \mathrm{AU}$ and its aphelion from $\sim 8 \mathrm{AU}$ to $\sim 6 \mathrm{AU}$ (Carusi et al. 1985). At the time of the target selection by the mission (October 2007), the activity pattern of the comet was relatively unknown. From ISOPHOT data, Colangeli et al. (1999) obtained a $\mathrm{H}_{2} \mathrm{O}$ and $\mathrm{CO}_{2}$ production 
rates of $(3.1 \pm 0.2) \times 10^{28} \mathrm{~s}^{-1}$ and $(2.5 \pm 0.3) \times 10^{27} \mathrm{~s}^{-1}$, respectively, on January 1,1998 . This leads to a $\mathrm{CO}_{2} / \mathrm{H}_{2} \mathrm{O}$ abundance ratio of $\sim 8 \%$, showing that $103 \mathrm{P} /$ Hartley 2 is a relatively $\mathrm{CO}_{2}$ abundant comet with respect to several other comets. Also, from data obtained with the instrument SWS on board ISO, Crovisier et al. (2000) report measurements on water rotational temperature, averaged in field of view of $\sim 20^{\prime \prime}$ and the ortho-to-para ratio of $\sim 2.7$, which corresponds to a spin temperature of about $35 \mathrm{~K}$. The effective radius of the comet was first estimated as $0.71 \pm 0.13 \mathrm{~km}$ (Groussin et al. 2004), giving rise to an active fraction of $\sim 1$ at perihelion and $0.30 \pm 0.11$ at 1.11 AU postperihelion. Later on, Lisse et al. (2009) could determine a comet nucleus radius of $0.57 \pm 0.08 \mathrm{~km}$, whereas Snodgrass et al. (2010) also reports a size estimate of $\leq 1 \mathrm{~km}$ from data taken in 2008 . Likewise, Lisse et al. (2009) claims that under circumstances of small size and high activity, the comet might suffer strong non-gravitational forces such that the spin state might undergo substantial changes in the instantaneous spin rate, but also the direction of the spin pole might change by as much as $\sim 30^{\circ}$ in apex in a single perihelion passage.

An extensive monitoring of the comet activity long before, during, and after the fly-by has taken place all around the world (Meech et al. 2011). The aims were to characterize the comet activity pattern as the comet approached perihelion, to perform ground-based simultaneous observations with the mission fly-by and to put in context a large-scale gas and dust coma with the detailed nucleus and near-nucleus environment observed by the instruments on board the EPOXI mission.

In this article, we exploit the optical data acquired at the time of the comet perihelion, from the October 27 to the 29, 2010 , to analyse the gas and refractory component of the coma. The observations and data reduction are explained in Sect. 2; the data analysis, our results and their comparison with others currently available are given in Sect. 3; and the summary appears in Sect. 4.

\section{Observations and data reduction}

The comet was observed from the Observatorio El Roque de los Muchachos on October 27 and 29, 2010 with the 4.2-m William Herschel Telescope (WHT) of the Isaac Newton Group (ING). We used the instrument ACAM (aux-port camera), which is mounted permanently at a folded-Cassegrain focus of the telescope. The CCD is a deep depletion device with additional fringe suppression and an AR coating peaking at $900 \mathrm{~nm}$. The pixel size is $15 \mu \mathrm{m}$, which gives $0.25^{\prime \prime} /$ pixel.

ACAM can be used for imaging with a circular field of view with $8.3^{\prime}$ diameter and for low-resolution spectroscopy over the spectral range $\sim 350-940 \mathrm{~nm}$ with a resolution of $\sim 0.33 \mathrm{~nm} /$ pixel. The performance of the instrument is extraordinary, however scattered light from the Moon represents a severe problem when it is close to the zenith or when the observations are acquired within $\sim 25^{\circ}$ of it. Unfortunately, during the comet perihelion, the Moon was at $13^{\circ}, 1^{\circ}$ and $10^{\circ}$ from the comet on October 27, 28, and 29, 2010 respectively. The scattered moonlight typically manifests itself as a broad bright feature covering most of one half of the imaging area, and it has intensity a factor 2-3 times higher than on the uncontaminated imaging area. Therefore, it was impossible to acquire any useful data of $103 \mathrm{P} /$ Hartley on October 28, whereas during the other two nights the target had to be placed near the edges of the CCD to avoid the scattered moonlight contaminated area placed to the west (i.e. in the tail direction) as much as possible on October 27 and to the east (i.e. in the Sun direction) on October 29. Also, on
October 29, there was a technical problem with one of the filter wheels such that no broadband images could be acquired.

Images of comet 103P/Hartley 2 were obtained with Sloan-Gunn $R, I, G$ and Bessel $U$ filters, and narrowband filters centred on strong comet gas emission bands $\left(\mathrm{CN}, \mathrm{C}_{3}, \mathrm{C}_{2}\right.$ and $\mathrm{NH}_{2}$ ) and on clear continuum regions in blue (BC, 443.0/3.5 nm) and red (RC, 684.0/7.7 nm) wavelength ranges. We took consecutive series of five and three images with the broad- and narrowband filters, respectively. Appropriate bias, dark, and flat field frames were also taken each night. There were sky photometric conditions with a typical mean seeing $\sim 0.7-1.0^{\prime \prime}$ FWHM of the PSF during the observing campaign, excluding a sudden and brief change in the weather conditions on October 29 around 04:00 UT when the humidity varied almost by a factor of 1.5 , the wind changed direction, and the seeing degraded to $2.8^{\prime \prime}$ FWHM. Photometric stars were observed at an airmass similar to the comet observations for absolute flux calibration of the broadband filters. In the case of the $\mathrm{CN}, \mathrm{C}_{3}, \mathrm{C}_{2}, \mathrm{BC}$, and $\mathrm{RC}$ narrowband cometary filters, we observed the spectrophotometric standard star GD71 (at similar airmass as the comet) for converting the counts on the detector into erg $\mathrm{cm}^{-2} \mathrm{~s}^{-1}$. To obtain gas $(\mathrm{CN}$, $\mathrm{C}_{3}$ and $\mathrm{C}_{2}$ ) images of the comet 103P, the continuum contribution in the gas filter images has been removed by making use of the continuum scaling factor (Korsun \& Jockers 2002) as in Lin et al. (2007). The continuum emission contributed $\sim 32 \%$ of the total signal in the $\mathrm{CN}$ filter passband, $\sim 28 \%$ in the $\mathrm{C}_{3}$ passband and $\sim 80 \%$ in the $\mathrm{C}_{2}$ passband.

Regarding the long-slit spectroscopy, the slit of the spectrograph was oriented along the E-W direction on October 29 giving dust and gas radial profiles in approximately the Sunantisolar $\left(\mathrm{PA}=276^{\circ}\right)$ direction. However, due to an error during the instrument configuration on October 27, the slit was positioned along the direction $70^{\circ}-250^{\circ}$ counted from north towards east. This provides us with radial profiles that deviate by $23^{\circ}$ from the antisolar direction $\left(\mathrm{PA}=273^{\circ}\right.$ ) (see Fig. 1). For absolute calibration, observations of the HZ 7 spectrophotometric standard star were acquired. For the comet observations, the slit width was $2^{\prime \prime}$, whereas the usable selected length is $5.5^{\prime}$, which provides us with radial profiles along selected directions up to cometocentric projected distances of $\sim 15000 \mathrm{~km}$ in each direction. The spectrophotometric standard star was observed with a width of $5^{\prime \prime}$ and the same slit length. All comet observations were done with telescope tracking at the comet's proper motion. Table 1 lists the details of the observations acquired during October 27 and 29, 2010. The spectrum on October 29 was acquired at the time when weather dramatically varied for about one hour during that night.

Since the gas emission of 103P/Hartley 2 covers the entire length of the slit, the sky subtraction has been estimated from the edges of the frame in continuum regions. More details on the images and spectra reduction and calibration can be found in Lara et al. (2001, 2011), Bertini et al. (2009) and Lin et al. (2007, 2009).

The broad- and the BC and RC narrowband images have been used to study (i) the dust coma morphology by applying the radial renormalization (A'Hearn et al. 1986) and the LarsonSekanina method (Larson \& Sekanina 1984); (ii) the azimuthally averaged profile of surface brightnesses; (iii) Af $\rho$, and (iv) the dust colour maps as a function of projected cometocentric distance $\rho$ and of time during the perihelion. The $\mathrm{CN}, \mathrm{C}_{3}$, and $\mathrm{C}_{2}$ comet images have provided us with gas coma morphology, whereas the $\mathrm{NH}_{2}$ images were only considered for an initial inspection of the coma since no observations of the calibration star 

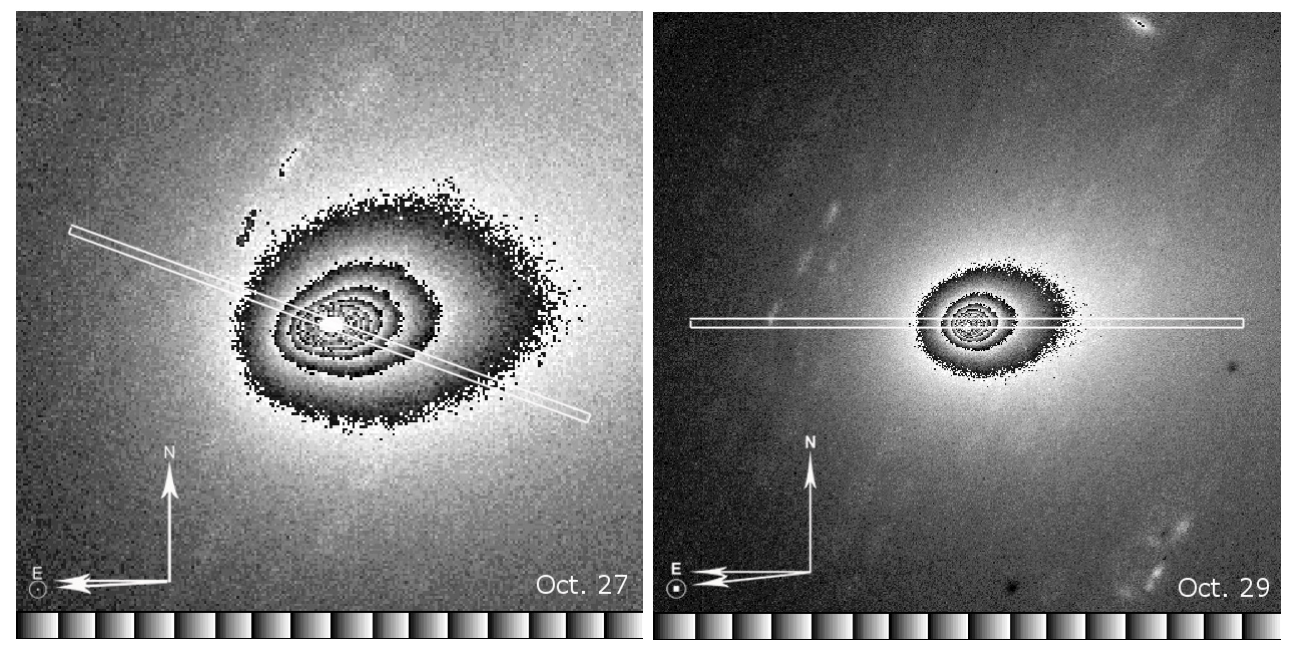

Fig. 1. Images of $103 \mathrm{P} /$ Hartley 2 on October 27 and 29, 2010 on which we have superimposed an imaginary slit to show the directions for which dust and gas radial profiles are obtained during the spectroscopic observations.

Table 1. Log of observations.

\begin{tabular}{|c|c|c|c|c|c|c|}
\hline $\begin{array}{l}\text { Date } \\
2010\end{array}$ & $\begin{array}{c}r_{\mathrm{h}}{ }^{a} \\
(\mathrm{AU})\end{array}$ & $\begin{array}{c}\Delta^{a} \\
(\mathrm{AU})\end{array}$ & $\begin{array}{l}\mathrm{PA}^{b} \\
(\mathrm{deg})\end{array}$ & $\begin{array}{c}\alpha^{c} \\
(\mathrm{deg})\end{array}$ & UT & Filter \\
\hline \multirow[t]{14}{*}{ October 27} & 1.0588 & 0.1281 & 272.4 & 56.6 & $00: 12-00: 25$ & Sloan-Gunn R \\
\hline & & " & $"$ & " & $00: 25-00: 31$ & Sloan-Gunn I \\
\hline & & " & " & " & $00: 34-00: 38$ & Sloan-Gunn G \\
\hline & & " & " & " & $00: 45-00: 57$ & Bessel U \\
\hline & & " & " & " & $01: 05-01: 13$ & $\mathrm{NH}_{2}$ \\
\hline & & 0.1282 & $"$ & " & 01:39-01:42 & $\mathrm{RC}$ \\
\hline & & " & " & " & $01: 44-01: 49$ & $\mathrm{BC}$ \\
\hline & & 0.1283 & " & " & $01: 53-02: 13$ & $\mathrm{C}_{2}$ \\
\hline & & " & 272.5 & " & $02: 34-02: 55$ & $\mathrm{CN}$ \\
\hline & & " & 272.6 & " & $03: 08-03: 13$ & $\mathrm{C}_{2}$ \\
\hline & & 0.1284 & " & " & $03: 16-03: 27$ & $\mathrm{BC}$ \\
\hline & & 11 & " & " & $03: 33-03: 35$ & $\mathrm{RC}$ \\
\hline & & 0.1285 & 272.7 & 56.7 & 04:49 & V400 (spectrum) \\
\hline & & 0.1286 & 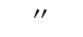 & 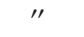 & $06: 15-06: 30$ & $\mathrm{CN}$ \\
\hline \multirow[t]{9}{*}{ October 29} & 1.0587 & 0.1333 & 275.8 & 57.5 & $01: 11-01: 43$ & $\mathrm{CN}$ \\
\hline & & 0.1334 & 275.9 & " & $02: 30-02: 38$ & $\mathrm{C}_{3}$ \\
\hline & & " & " & " & $02: 43-02: 51$ & $\mathrm{C}_{2}$ \\
\hline & & " & 276.0 & " & $02: 55-03: 10$ & $\mathrm{C}_{3}$ \\
\hline & & 0.1335 & " & $"$ & 03:19-03:34 & $\mathrm{BC}$ \\
\hline & & " & " & " & $03: 42-03: 35$ & $\mathrm{RC}$ \\
\hline & & 0.1336 & " & " & 04:01 & V400 (spectrum) \\
\hline & & 0.1337 & 276.1 & " & $04: 56-05: 35$ & $\mathrm{CN}$ \\
\hline & & 0.1338 & & " & $05: 45-05: 57$ & $\mathrm{BC}$ \\
\hline
\end{tabular}

Notes. ${ }^{(a)}$ The heliocentric and geocentric distance of the component during the observations, respectively; ${ }^{(b)}$ the position angle of the extended Sun-target radius vector as seen in the observer's plane-of-sky, measured counter clockwise (east) from reference frame, North Celestial Pole;

(c) the phase angle Sun-comet-observer.

GD71 were acquired with this filter, and thus no flux calibration has been done for the $\mathrm{NH}_{2}$ images.

On the other hand, the spectra have provided us with the production rates for $\mathrm{CN}, \mathrm{C}_{3}, \mathrm{C}_{2}$ and $\mathrm{NH}_{2}$ in the context of the Haser model (Haser 1957), with dust brightness profiles as a function $\rho$ along the directions imposed by the slit (see Fig. 1), with dust colour variations in those same directions and with $A f \rho$ in different continuum spectral ranges.

\section{Data analysis and results}

\subsection{Dust}

The analysis of the dust coma of comet 103P/Hartley 2 was carried out from the images and the spectra. This analysis comprises: coma morphology, dust production rate through the Af $\rho$ parameter, and dust colour from the $2 \mathrm{D}$ images and from the long-slit spectra.

To determine whether some morphological structures are present in the coma of $103 \mathrm{P} /$ Hartley 2 , we investigated all of the flux-calibrated images acquired, either in broadband filters as in cometary narrowband filters with bandpass centred on clear continuum regions, that is, 443 (BC) and $684 \mathrm{~nm}(\mathrm{RC})$. From a first inspection, the green and red broadband images clearly display the dust+gas coma. The bandpass of the $\mathrm{G}$ and R Sloan-Gunn filters do contain strong gas emission in $\mathrm{C}_{2}, \mathrm{NH}_{2}$, and $\mathrm{H}_{2} \mathrm{O}^{+}$. On the other hand, the comet images acquired with the bandpass of the I Sloan-Gunn filter show much less gas contamination, although fainter cometary gas emissions (the $\mathrm{CN}$ red system) also fall in the bandpass. Figure 2 shows the comet images in G, R, and I Sloan-Gunn together with their radially renormalized frames (A'Hearn et al. 1986) in each filter. Aside from a 

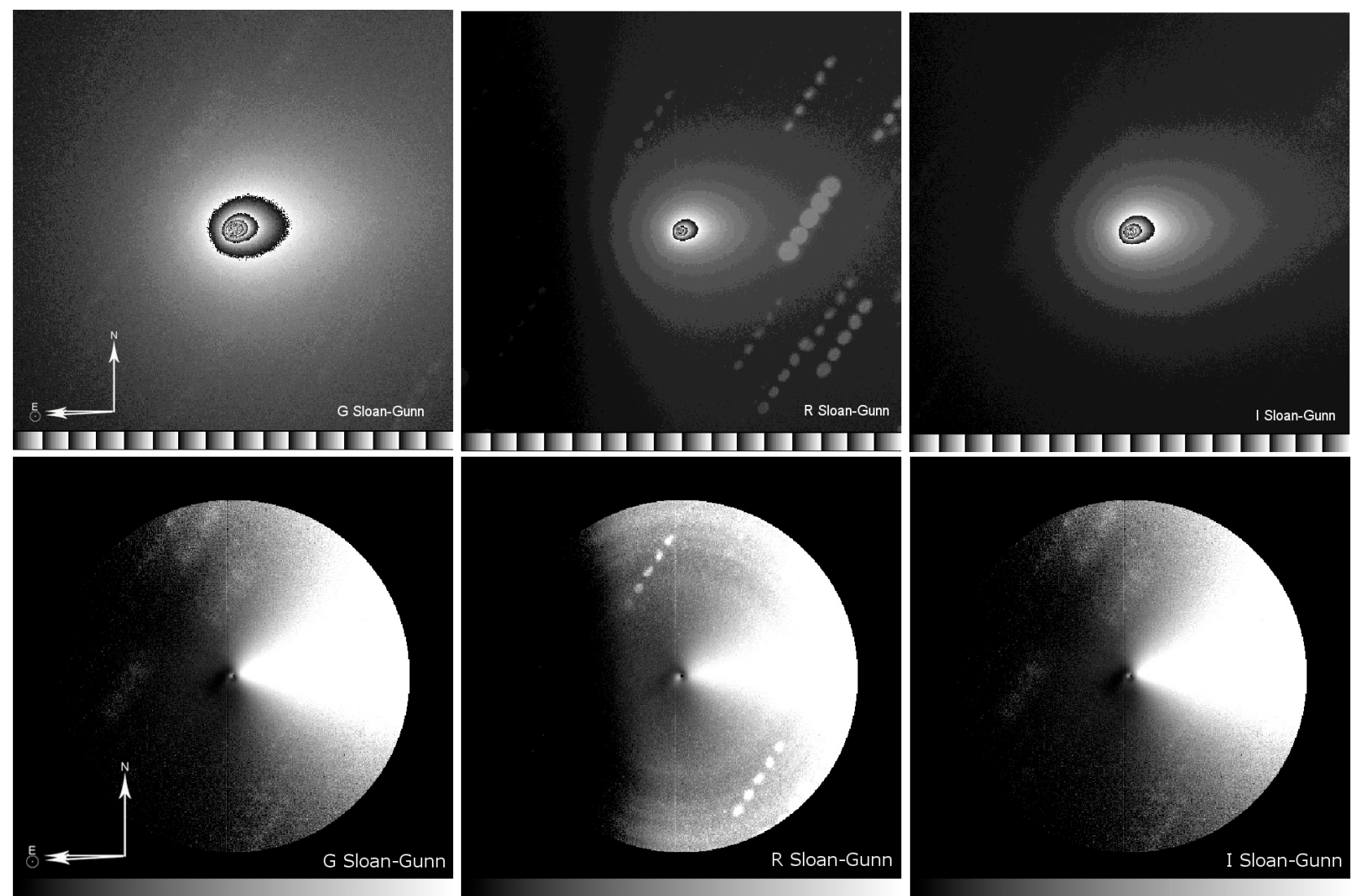

Fig. 2. Images of 103P/Hartley 2 on October 27, 2010, acquired with $G, R$ and $I$ Sloan-Gunn filters (from left to right), together with their corresponding radially renormalized images to enhance the structures in the coma. The FOV is $125^{\prime \prime} \times 125^{\prime \prime}$, meaning $11600 \times 11600 \mathrm{~km}$ at the comet's distance.

clear dust tail, no evident feature can be detected in these images except in the one obtained with $\mathrm{R}$ broadband where a faint jet seems to be detected in Sun direction.

Therefore, a meaningful morphological analysis of the dust coma has been done on the flux-calibrated images acquired with the $\mathrm{BC}$ and $\mathrm{RC}$ cometary filters. Figure 3 displays the dust coma of 103P on October 27 and 29 imaged with the BC narrowband filter, the $\mathrm{RC}$ one, the quotient of the images in $\mathrm{BC}$ and $\mathrm{RC}$ taken a few hours apart, and the radially renormalized $\mathrm{RC}$ images.

A detailed inspection of the processed images shows that, beside the dust tail, on October 27 at 01:41 UT a faint jet in the sunward direction starts to be visible in the red continuum (see Fig. 4), becoming stronger and more obvious in the images acquired approximately two hours later with both the BC and RC narrowband cometary filters.

On October 27, both for the blue and red continuum, the quotient image (third column in Fig. 3) shows a clear dust structure pointing towards the Sun and slightly curved to the south. This feature is not clearly detected on October 29. Both the dust tail and the curved jet pointing to the Sun are also enhanced by means of the Larson-Sekanina method (Larson \& Sekanina 1984). An independent verification can be done by looking the anisotropies in the isophotes (first two columns in Fig. 3). The turn on/off of the curved jet in the eastern direction has been explained as the response of an active area that faces the Sun coinciding with the nucleus spin period (Harmon et al. 2011) during October 24-27, 2010. This finding in our data is confirmed by the EPOXI Team when analysing a sequence of images acquired by the HRI and MRI instruments on board. Assuming that on October 27 between 01:42 and 03:33 this active area faced the
Sun and that the nucleus spin period is $18.1 \mathrm{~h}$ (Harmon et al. 2011), the dust coma of 103P would have displayed the same structure on October 29 at $\sim 07: 30$ UT i.e. during dawn at the Observatorio El Roque de los Muchachos but unfortunately was not observable.

From the set of images and spectra on October 27 and 29, we have characterized the dust coma as much as possible in terms of dust colour and production rates $(A f \rho)$, and of surface brightness radial profiles. Figure 5 shows the maps of dust reddening in \%/100 nm for October 27 and 29, computed following the relation (Turner \& Smith 1999)

colour $=\frac{\mathrm{RC}-\mathrm{BC}}{6.84-4.43} \frac{200}{\mathrm{BC}+\mathrm{RC}}$

where $\mathrm{RC}$ and $\mathrm{BC}$ refer to the comet images acquired with the red and blue narrowband cometary filters (centred at $684.0 \mathrm{~nm}$ and at $443.0 \mathrm{~nm}$, respectively) after removing the solar contribution. The average dust reddening in a circular aperture of $\sim 500 \mathrm{~km}$ of radius is $\sim 24 \% / 100 \mathrm{~nm}$ for both dates, a rather common value of cometary dust colour. This behaviour of the dust colour seems to indicate that the optical properties of the dust grains do not significantly vary regardless of where they are measure in the jet or in the surrounding coma. However, it is interesting that the dust in the jet has different optical properties when compared with the "older" dust in the background coma (see bottom right in Fig. 5). Comparison of the dust coma in the $\mathrm{RC}$, when the jet is already clearly visible in the coma, with the dust coma in the $\mathrm{BC} 2 \mathrm{~h}$ before, shows dust reddening variations. We measure normalized dust reflectivity of 45\%/100 nm at the optocentre decreasing to $24 \% / 100 \mathrm{~nm}$ at the outer edge 

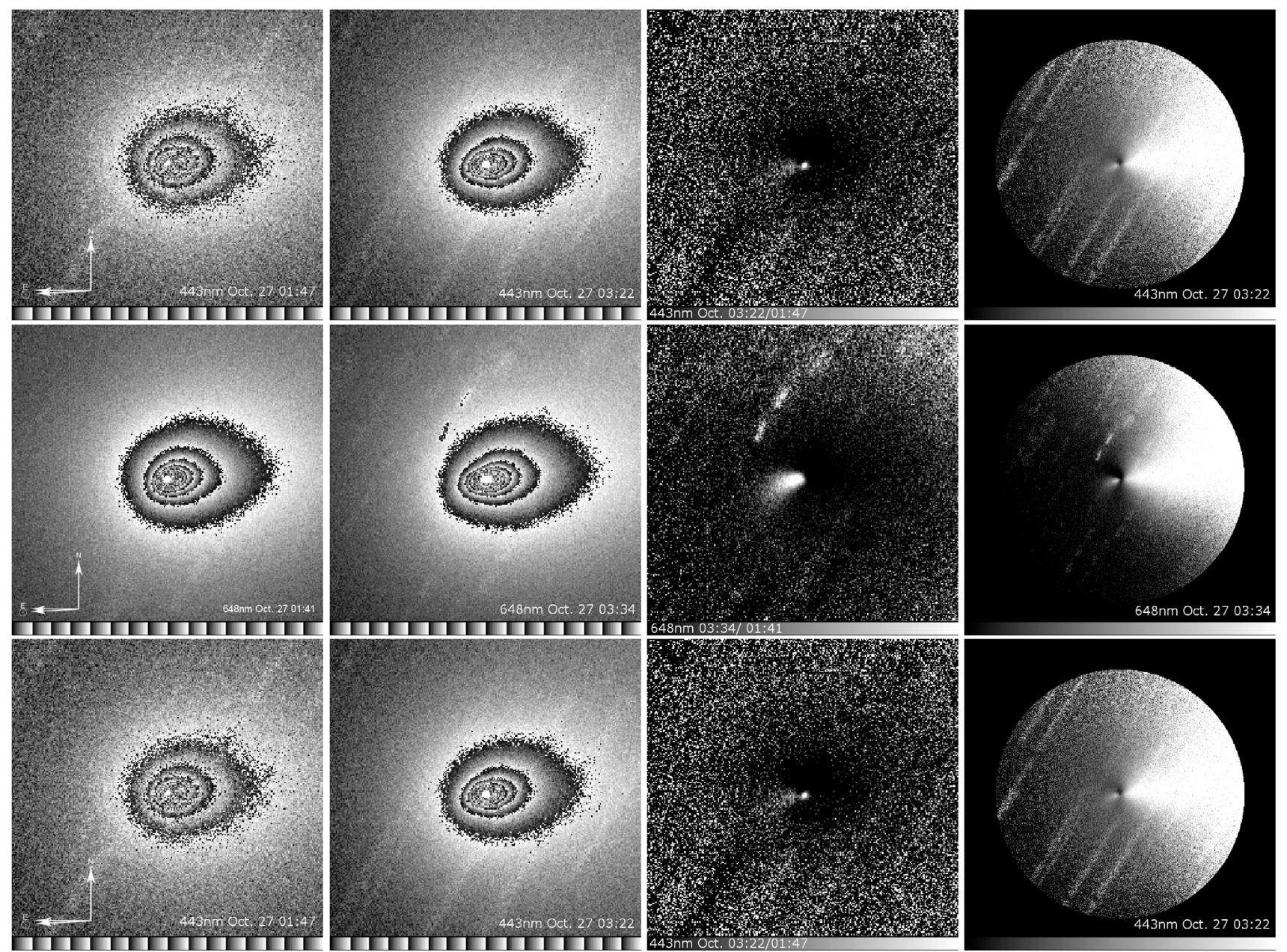

Fig. 3. Flux-calibrated images of 103P/Hartley 2 in a look-up table to display the isophote anisotropies. The images are acquired with clear continuum filters centred at $443 \mathrm{~nm}$ with cuts between 0 and $2 \times 10^{-15} \mathrm{erg} \mathrm{cm}^{-2} \mathrm{~s}^{-1}$, and at $684 \mathrm{~nm}$ with cuts between 0 and $8 \times 10^{-15} \mathrm{erg} \mathrm{cm}^{-2} \mathrm{~s}^{-1}$. The third column shows the quotient of the comet images obtained a few hours apart (i.e. 2nd column/1st column) with cuts between 1 and 1.6. The fourth column displays the radially renormalized images of the ones in the second column. The FOV is $62.5^{\prime \prime} \times 62.5^{\prime \prime}$, meaning $5800 \times 5800 \mathrm{~km}$ at the comet's distance.

$(\sim 840 \mathrm{~km})$ along the jet axis. The origin of this colour gradient along the jet axis can be explained as the consequence of comparing old processed dust and new refractory material dragged by the outgassing when the active area is exposed to the Sun. This higher reddening around the optocentre characterizes this new material freshly expelled from the comet nucleus. These refractory particles get bluer while travelling outwards, meaning that there are variations in the optical properties caused either by fragmentation, ice sublimation, or both.

The long-slit spectra cover a wide wavelength range and they contain spatial information in several directions. Therefore, the dust colour and its variation with $\rho$ can be determined from these measurements, too. Several band passes representing continuum emissions are $415.0-417.5 \mathrm{~nm}, 440.0-437.0 \mathrm{~nm}$, $483.3-489.0 \mathrm{~nm}, 522.0-530.0 \mathrm{~nm}, 677.5-692.5 \mathrm{~nm}$, and $680.0-686.0 \mathrm{~nm}$. The comet spectra have been divided by the solar spectrum (Kurucz et al. 1984), degraded to the same spectral resolution as the ACAM data to remove the solar colour when computing the cometary dust colour. Normalized reflectivity, or dust reddening, is computed as in Eq. (1) where we substitute $\mathrm{BC}$ and $\mathrm{RC}$ for different combinations of the blue and red spectral ranges mentioned above in wavelength regions where differences in sky colour are not dramatically high. Figure 6 shows the continuum reflectivity slope for October 27 and 29, 2010 along the directions dictated by the slit positioning (see Sect. 3 and Fig. 1). We have only considered the innermost coma, i.e. $-1500 \leq \rho \leq 1500 \mathrm{~km}$, to investigate the dust colour reddening from the spectroscopic measurements, because the scattered moonlight which falls in the slit length poses a severe problem at distances $\rho \geq 2000 \mathrm{~km}$ from the optocentre. For both dates, we see that dust colour gets considerably bluer in the sunward hemisphere, whereas it can be considered constant in approximately tail direction. The absolute values of this dust reddening depend on the spectral ranges, but the trend explained above is confirmed for all of them (see Fig. 6).

Table 2 lists the slope of the surface brightness radial profiles for both October 27 and 29, and Fig. 7 shows the dust surface brightness profiles in the direction at PA $70-250^{\circ}$ and in E-W direction (PA 90-270 ), respectively. The surface brightness $B$ is computed from the flux-calibrated continuum profiles multiplied by $2 \pi \Delta^{2}$ and $r_{\mathrm{H}}^{2}$ and normalized to a unit area of $1 \mathrm{~km}^{2}$ at the position of the comet. This leads to a surface brightness in units of energy per second per square kilometer per angstrom (erg s${ }^{-1} \mathrm{~km}^{-2} \AA^{-1}$ ), which is independent of the geocentric distance, as well as of the size of the aperture and normalized to the solar flux at $r_{\mathrm{H}}=1 \mathrm{AU}$. It therefore allows a direct comparison of the day-to-day variations in the continuum profiles. The results in Table 2 and in Fig. 7 support the idea that the 


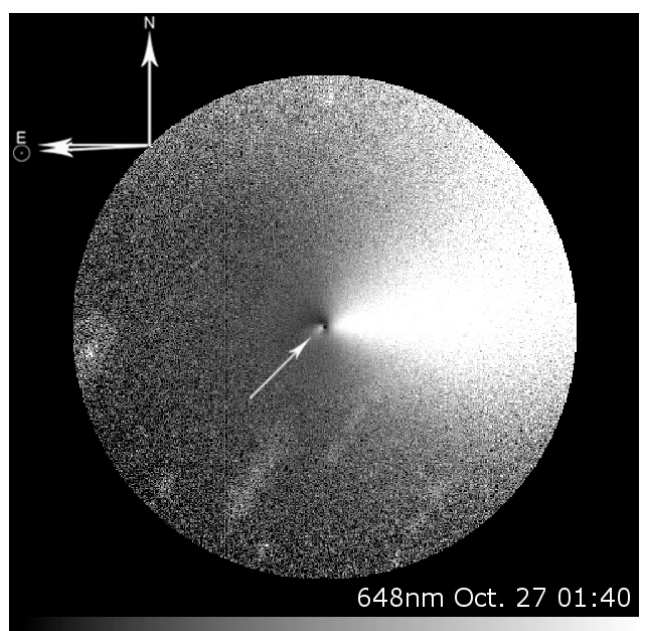

Fig. 4. Radially renormalized image of comet 103P/Hartley 2 obtained on October 27, 2010 at $\sim 01: 40$ UT with the RC cometary filter. The arrow points to the faint jet that starts to be visible at that time. Its intensity increases two hours later as clearly visible in Fig. 3. The FOV is $62.5^{\prime \prime} \times 62.5^{\prime \prime}$, meaning $5800 \times 5800 \mathrm{~km}$ at the comet's distance.

feature in the sunward hemisphere was still detectable in the coma of 103P/Hartley 2 about four hours after its activation at 01:00 UT according to the EPOXI Team (see http://epoxi . umd.edu/3gallery/20101027-jets.shtml) since our data were acquired at $\sim 05: 00$ UT on October 27, 2010. The similarity between the $\log B$ versus $\log \rho$ profiles on October 27 and 29 also indicates that the jet was present in the eastern hemisphere on October 29 at 04:01 UT, although this cannot be confirmed through dust images since none was acquired after the spectrum acquisition (see Table 1).

The release of dust from the comet is approximately determined through optical measurements of the parameter $A f \rho(\mathrm{cm})$ as a function of the projected cometocentric distance $\rho$ (A'Hearn et al. 1984)

$A f \rho=\frac{\left(2 \Delta r_{\mathrm{h}}\right)^{2}}{\rho} \frac{F_{\mathrm{c}}}{F_{\mathrm{S}}}$

where $\Delta$ (in $\mathrm{cm}$ ) and $r_{\mathrm{h}}$ (in $\mathrm{AU}$ ) are the comet's geocentric and heliocentric distances, respectively, $F_{\mathrm{c}}$ is the measured cometary flux in the selected filter (narrowband cometary filters BC and $\mathrm{RC}$ ) integrated in the radius of aperture $\rho$, and $F_{\mathrm{S}}$ is the total solar flux in the same filter.

Table 3 contains the Af $\rho$ values measured on October 27 and 29, 2010 from the BC and RC comet images (see Table 1), as well as from the spectroscopic measurements in three different continuum regions. In the case of the images, we considered a circular aperture of radius $\rho \sim 4700 \mathrm{~km}$. The parameter $A f \rho$ in the blue and red continuum is 59 and $102 \mathrm{~cm}$, respectively, on October 27, and 56 and $111 \mathrm{~cm}$ on October 29. The dust colour reddening computed from these two values of $A f \rho$ is $\sim 22 \% \mathrm{~nm}$, in good agreement with results from spectroscopic data. Regarding the spectroscopic measurements, Af $\rho$ can also be computed in several continuum regions (see Table 2). For this, we have determined the equivalent radius of the aperture provided by the long-slit (width $=2^{\prime \prime}$, useful length $=330^{\prime \prime}$, pixel size $=0.25^{\prime \prime}$ ) according to the expression given in Roettger (1991), yielding 4.329" ( 400 km). The results are also listed in Table 3 on both dates. While there is an overall agreement between the Afo computed from the $\mathrm{BC}$ and $\mathrm{RC}$ images and the long-slit spectrum on October 27, this not the case for October
29 when the long-slit spectrum generally provides much lower Af $\rho$ values than two days before and also noticeably different in the BC range. Since the comet activity variations reported by Biver et al. (2010, priv. comm.) do not reach a factor of $\sim 2$, we have checked the weather conditions at the time of the spectrum acquisition. Unfortunately, the humidity increased to $\sim 50 \%$, the wind dramatically changed direction and the seeing increased to $2.8^{\prime \prime}$. Therefore, the reason for such a discrepancy most likely lies in the unstable weather conditions during data acquisition and not on intrinsic comet activity variability.

\subsection{Gas}

Although the signal-to-noise ratio of the data acquired with the gas narrowband cometary filters is not very high, we have investigated the general spatial distribution of gas species in the coma of comet 103P/Hartley 2 in the $\mathrm{CN}, \mathrm{C}_{2}$, and $\mathrm{C}_{3}$ images.

In $\mathrm{CN}$ and $\mathrm{C}_{3}$, the coma is noticeably asymmetric on both dates, showing a north-south elongation with clear differences between October 27 and October 29, 2010 in the case of CN. The first two frames in Fig. 8 show the $\mathrm{CN}$ image after processing with the Larson-Sekanina technique. It is clear that on October 27 , there is a more pronounced over abundance $(\sim 10 \%)$ of $\mathrm{CN}$ particles in the southern hemisphere (as projected on the plane of the sky), whereas on October 29, both cones in the northern and southern hemispheres display similar over abundances $(\sim 5 \%)$ when compared to the isotropic gas coma. These variations in the $\mathrm{CN}$ jet appearance are related to the spin period of the comet nucleus as already reported by Samarasinha et al. (2011). On October 29, we detected that the $C_{3}$ coma is also asymmetric, displaying two cones in the north-south direction, centred on the nucleus, where the column density is enhanced with respect to any other direction. This behaviour is rather similar to the one seen in $\mathrm{CN}$. No $\mathrm{C}_{3}$ data were acquired on October 27, therefore we cannot conclude whether a similar anisotropic $\mathrm{C}_{3}$ coma already existed. On the other hand, the $\mathrm{C}_{2}$ gas coma does not display any evident asymmetry.

The acquired spectra of the comet are used to investigate the $\mathrm{CN}, \mathrm{C}_{3}, \mathrm{C}_{2}$, and $\mathrm{NH}_{2}$ column density profiles in the directions imposed by the slit positioning, that is, in the $70^{\circ}-250^{\circ}$ direction from north towards east on October 27 and in E-W direction on October 29, 2010. Figure 9 shows the flux-calibrated spectrum of comet 103P/Hartley 2 acquired on October 27, 2010 where the strongest cometary emissions in the optical range $\left(\mathrm{CN}, \mathrm{C}_{2}\right.$, $\mathrm{C}_{3}$, and $\mathrm{NH}_{2}$ ) are indicated. The slit was positioned as indicated in Fig. 1.

The spectral regions and the subtraction of the underlying continuum in the gas emission bands is done as described by Lara et al. (2001). The conversion of the emission band fluxes into column densities makes use of constant $g$-factors for $\mathrm{C}_{2}$, $\mathrm{C}_{3}$, and $\mathrm{NH}_{2}$ (A'Hearn et al. 1995), whereas the $g$-factor of the $\mathrm{CN}$ molecule is calculated for the heliocentric distance and velocity of 103P/Hartley 2 for both dates from the set of values given by Schleicher (1983). Although it has been reported that the gas production rate of $103 \mathrm{P}$ is not steady, showing variations in days and minutes Biver et al. (2010, priv. comm.), for giving any indication of the gas production rates, we computed them by means of the Haser modelling (Haser 1957) with parent velocity $v_{\mathrm{p}}$ scaled with $r_{\mathrm{h}}\left(v_{\mathrm{p}}=0.86 r_{\mathrm{h}}^{-0.4} \mathrm{~km} \mathrm{~s}^{-1}\right)$, customary values for the daughter velocity $v_{\mathrm{d}}=1 \mathrm{~km} \mathrm{~s}^{-1}$ and scale lengths given in A'Hearn et al. (1995). For the corresponding set of parameters in the Haser modelling, we produced theoretical column density profiles for each species by varying the production rate until the 

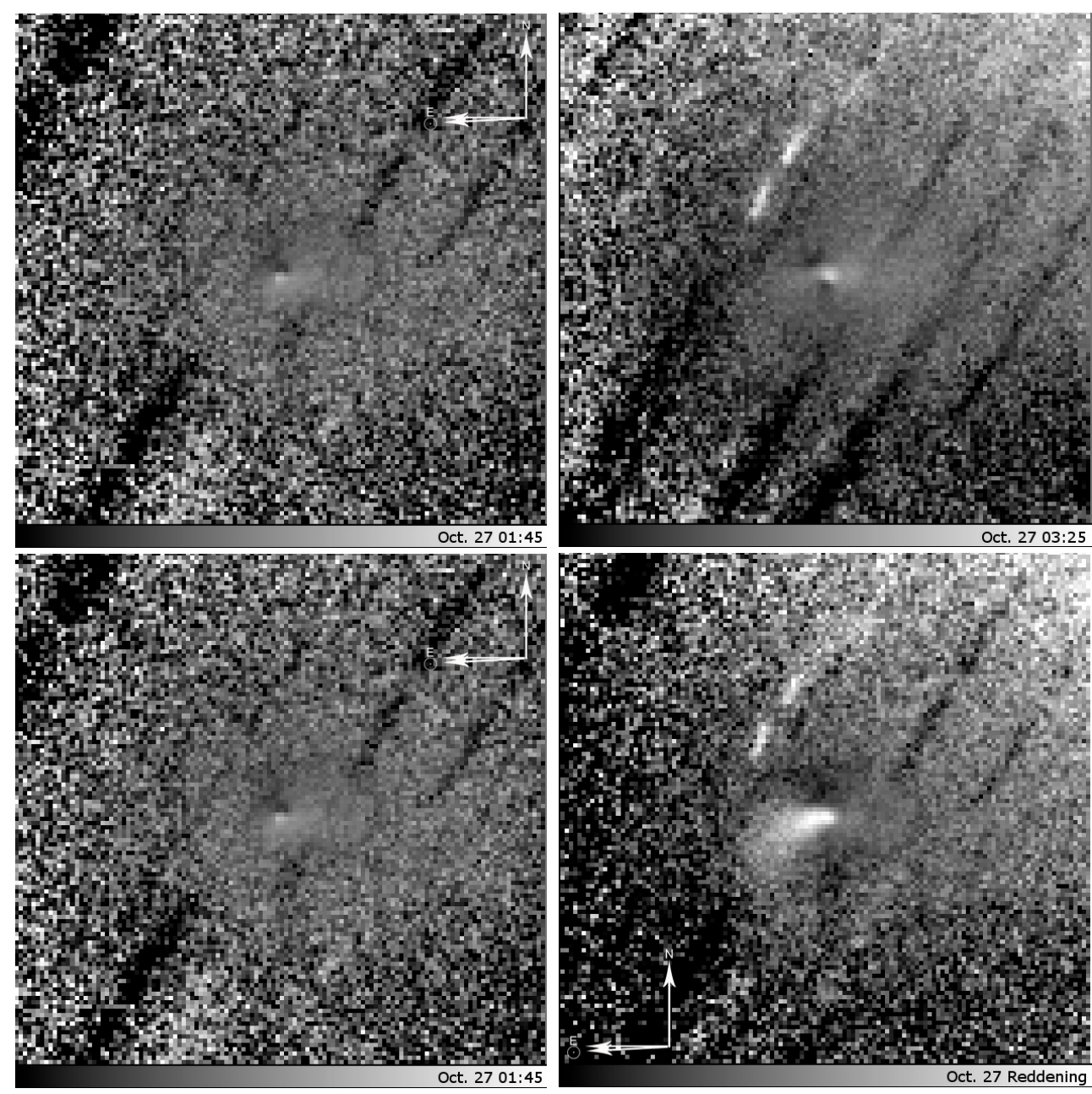

Fig. 5. Dust reddening, in $\%$ per $100 \mathrm{~nm}$, of the coma of 103P/Hartley 2 on October 27 and 29, 2010. Bottom right frame in the figure displays the dust reddening of the new material in the jet compared with the older dust in the coma on October 27. The look-up table is linear and stretches from 15 to $40 \% / 100 \mathrm{~nm}$.

Table 2. Linear fits to the $\log B-\log \rho$ profiles obtained from the long-slit spectroscopic measurements, giving the slope $m$.

\begin{tabular}{|c|c|c|c|c|c|c|}
\hline \multirow{2}{*}{$\begin{array}{l}\text { Date } \\
2010\end{array}$} & \multirow[t]{2}{*}{ Direction } & \multicolumn{5}{|c|}{ Continuum regions } \\
\hline & & $\begin{array}{l}482-485 \mathrm{~nm} \\
\text { cont } 1\end{array}$ & $\begin{array}{l}520-525 \mathrm{~nm} \\
\text { cont } 2\end{array}$ & $\begin{array}{c}\text { 680-690 nm } \\
\text { cont3 }\end{array}$ & $\begin{array}{l}443 \mathrm{~nm} \\
\mathrm{BC}\end{array}$ & $\begin{array}{l}684 \mathrm{~nm} \\
\mathrm{RC}\end{array}$ \\
\hline October 27 & $70^{\circ a}$ & -0.51 & -0.50 & -0.47 & & \\
\hline \multirow{4}{*}{ October 29} & $250^{\circ}$ & -1.09 & -1.19 & -1.80 & & \\
\hline & azimuthal average $^{b}$ & & & & $\begin{array}{l}-0.98 \\
-1.03\end{array}$ & $\begin{array}{l}-1.02 \\
-1.03\end{array}$ \\
\hline & east & -0.61 & -0.56 & - & & \\
\hline & $\begin{array}{c}\text { west } \\
\text { azimuthal average }{ }^{b}\end{array}$ & -1.07 & -1.12 & -1.32 & $\begin{array}{l}-0.98 \\
-0.96\end{array}$ & -0.94 \\
\hline
\end{tabular}

Notes. mSlope of the linear fit $\log B$ vs. $\log \rho$ for $2.0 \leq \log \rho \leq 4$ (i.e. $100 \leq \rho \leq 10000 \mathrm{~km}$ ). Standard deviation of these fits is always lower than 5\%; (a) the fits in this direction have a considerably higher standard deviation, which is caused by the existence of the jet on October 27 ; ${ }^{(b)}$ a radius $\rho$ equal to $50^{\prime \prime}$ (i.e. $\sim 4700$ and $\sim 4800 \mathrm{~km}$ on October 27 and 29, 2010 respectively) has been used to compute the slope of the linear fit.

best match between observations and theoretical predictions was achieved.

The production rates, $Q_{i}$, led to a best match for the observed column densities, the quotient $\left[Q\left(\mathrm{C}_{2}\right) / Q(\mathrm{CN})\right]$, and $\log$ $\left[A f \rho(520-525 \mathrm{~nm}) / Q\left(C_{2}\right)\right]$ are listed in Table 3, whereas Figs. 10 and 11 show the observed $\mathrm{CN}, \mathrm{C}_{3}, \mathrm{C}_{2}$, and $\mathrm{NH}_{2}$ column density radial profiles in the directions probed by the slit, together with the best achievable Haser fit. The observed and theoreti- cal gas profiles in Figs. 10 and 11 show the complexity of fitting these profiles as the coma is rather asymmetric. The use of any model (either vectorial modelling (Festou 1981a,b) or timedependent one as considered by (Lara et al. 2001) for comet $1996 / Q 1$ Tabur) will be unable to reproduce the E-W asymmetries. Some improvement in the fits can be achieved when "timedependent" production rates are considered. For implementing this model, the production rate is doubled for $\mathrm{CN}$ (and to a 
A\&A 532, A87 (2011)
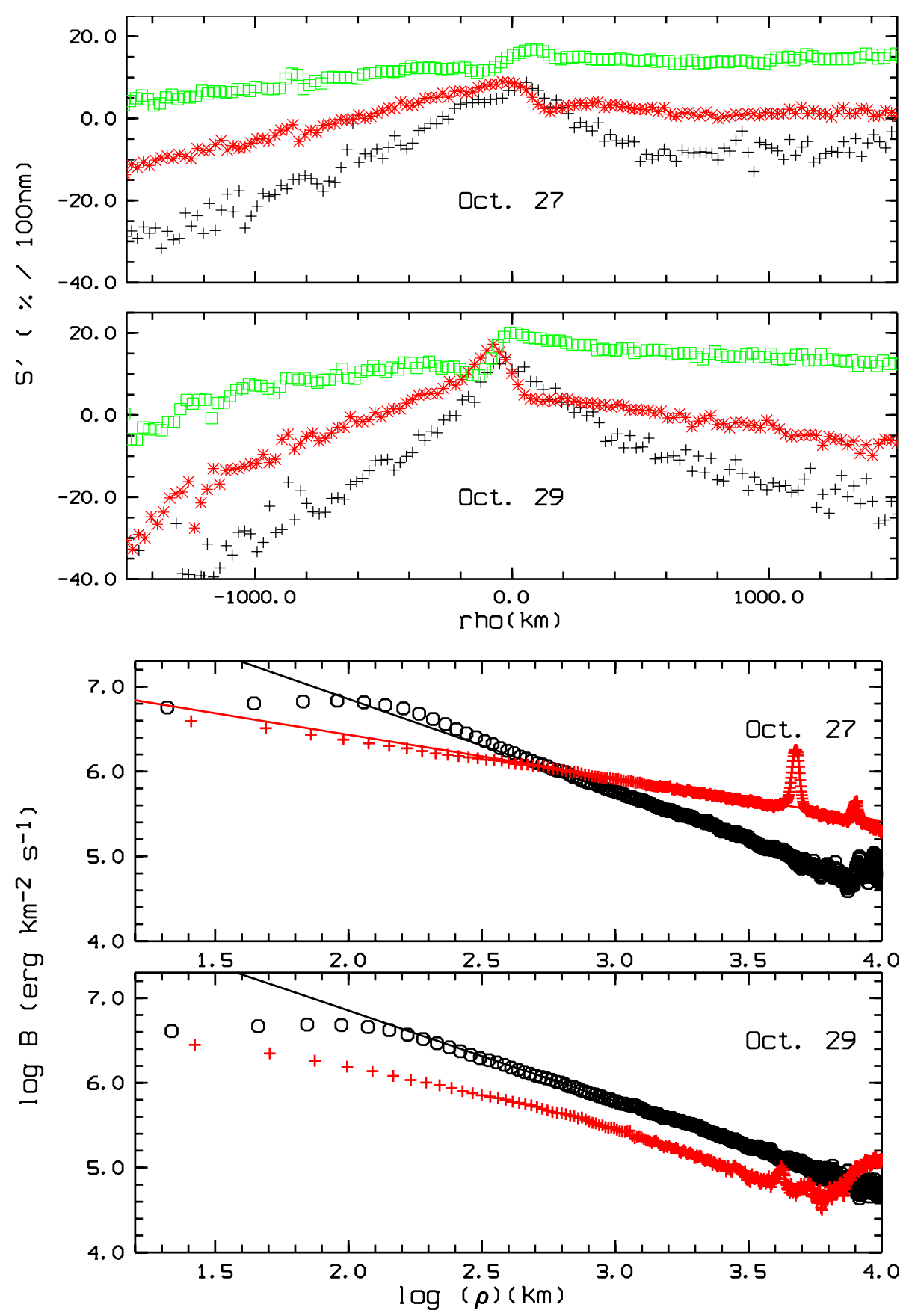

Fig. 6. Dust colour profiles, or normalized reflectivity $S^{\prime}$ in units of $\%$ per $100 \mathrm{~nm}$ as a function of projected cometocentric distance, $\rho$, on October 27 and 29, 2010 obtained from the spectroscopic measurements. Negative $\rho$ refers to the $70^{\circ}$ direction on October 27 and to east direction on October 29. Positive $\rho$ refers to the direction at $\mathrm{PA}=250^{\circ}$ on October 27 and to the westward direction on October 29. Black crosses: normalized reflectivity computed between the ranges $415.0-417.5 \mathrm{~nm}$ and 522.0-530.0 $\mathrm{nm}$. Red asterisks: normalized reflectivity computed between the ranges 483.3-489.0 $\mathrm{nm}$ and 680.0-686.0 nm. Green open squares: normalized reflectivity computed between the ranges $440.0-437.0 \mathrm{~nm}$ and $677.5-692.5 \mathrm{~nm}$, which are the same regions as the $\mathrm{BC}$ and $\mathrm{RC}$ filters.

Fig. 7. Dust surface brightness profiles $B$ as a function of projected cometocentric distance $\rho$ in double logarithmic representation, obtained from the spectroscopic measurements on October 27 and 29, 2010 in the tailwards (red crosses) - sunward (black open circles) directions. The selected continuum region is 482-485 $\mathrm{nm}$. Symbols refer to the observational data and a solid line refers to the linear fit.

Table 3. Dust ( $A f \rho$ ) and gas production rates of the comet 103P/Hartley 2.

\begin{tabular}{|c|c|c|c|c|c|c|c|c|c|c|c|}
\hline \multirow{2}{*}{$\begin{array}{l}\text { Date } \\
2010\end{array}$} & \multicolumn{4}{|c|}{$A f \rho(\mathrm{cm})$} & \multicolumn{5}{|c|}{$\operatorname{Gas}\left(\mathrm{s}^{-1}\right)$} & \multirow[b]{2}{*}[Q(\mathrm{C}_{2})/Q(\mathrm{CN})]{} & \multirow[b]{2}{*}{$\log [A f \rho / Q(\mathrm{CN})]$} \\
\hline & cont 1 & cont 2 & cont3 & $\mathrm{BC}$ & $\mathrm{RC}$ & $\mathrm{CN}$ & $\mathrm{C}_{3}$ & $\mathrm{C}_{2}$ & $\mathrm{NH}_{2}$ & & \\
\hline October 27 & & & & 59 & 102 & & & & & & \\
\hline \multirow[t]{2}{*}{ October 29} & 152 & 143 & 131 & $\begin{array}{l}58 \\
56\end{array}$ & $\begin{array}{l}105 \\
111\end{array}$ & $1.07 \times 10^{25}$ & $1.11 \times 10^{24}$ & $1.42 \times 10^{25}$ & $2.14 \times 10^{26}$ & 1.31 & -22.88 \\
\hline & 78 & 92 & 74 & 46 & & $9.95 \times 10^{24}$ & $7.36 \times 10^{23}$ & $1.38 \times 10^{25}$ & $1.55 \times 10^{26}$ & 1.38 & -23.03 \\
\hline
\end{tabular}

lesser extent for the other species) in a short fraction of a day, $\sim 0.1$, whereas the steady comet activity can be reproduced by using the values listed in Table 3. Since the step-function change in production rate is merely for computational ease, this result only confirms the rapid and periodical increase in the cometary activity.
Also, another problem is the short geocentric distance and the available slit length that has allowed us to measure the gas column densities up to projected cometocentric distances of only $10000 \mathrm{~km}$. This, together with an approximate sky subtraction, gives rise to observed profiles noticeably deviate from the Haser theoretical profiles beyond $\log (\rho) \approx 3.8$. For the species with 

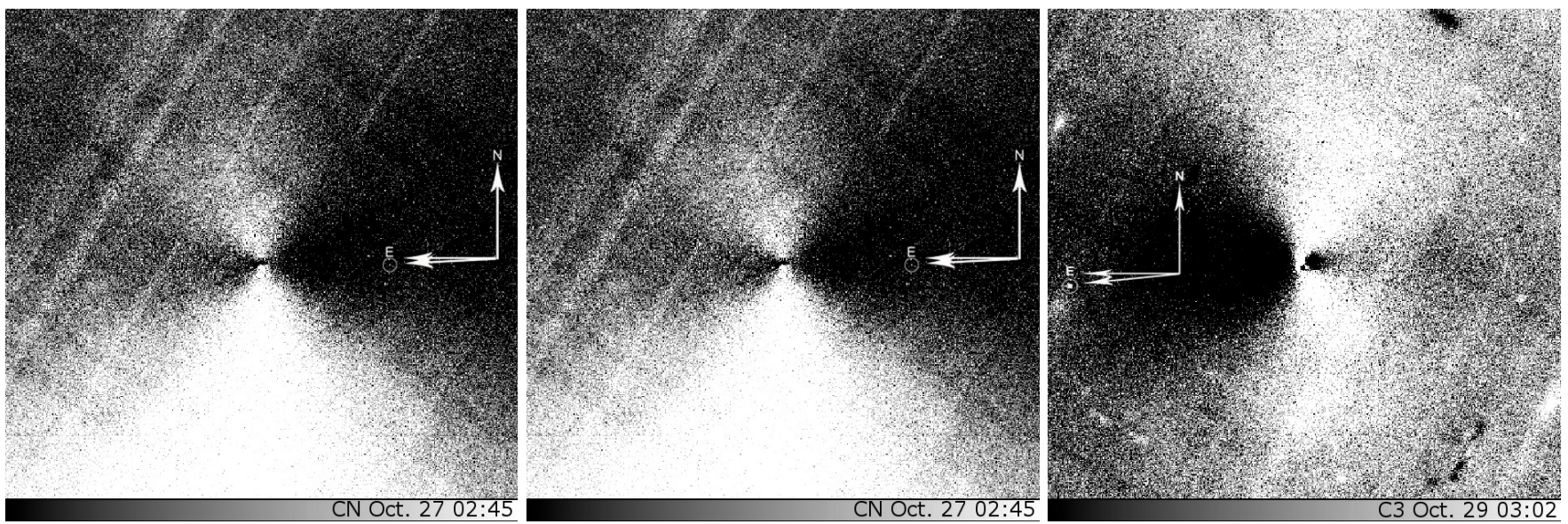

Fig. 8. Enhanced gas images of the comet obtained on October 27 and 29, 2010. The Larson-Sekanina method has been applied to fully calibrated images after subtracting the underlying continuum as explained in Sect. 2. From left to right: CN on October 27 at 02:45 UT (mid-time during exposure), $\mathrm{CN}$ on October 29 at 05:15 UT (mid-time during exposure), $\mathrm{C}_{3}$ on October 29 at 03:02 UT (mid-time during exposure). The FOV is $11800 \times 11800 \mathrm{~km}$. Aside from the reported CN jet by Samarasinha et al. (2011) and Meech et al. (2011), the $\mathrm{C}_{3}$ coma distribution also displays similar jet structures in the north-south direction.

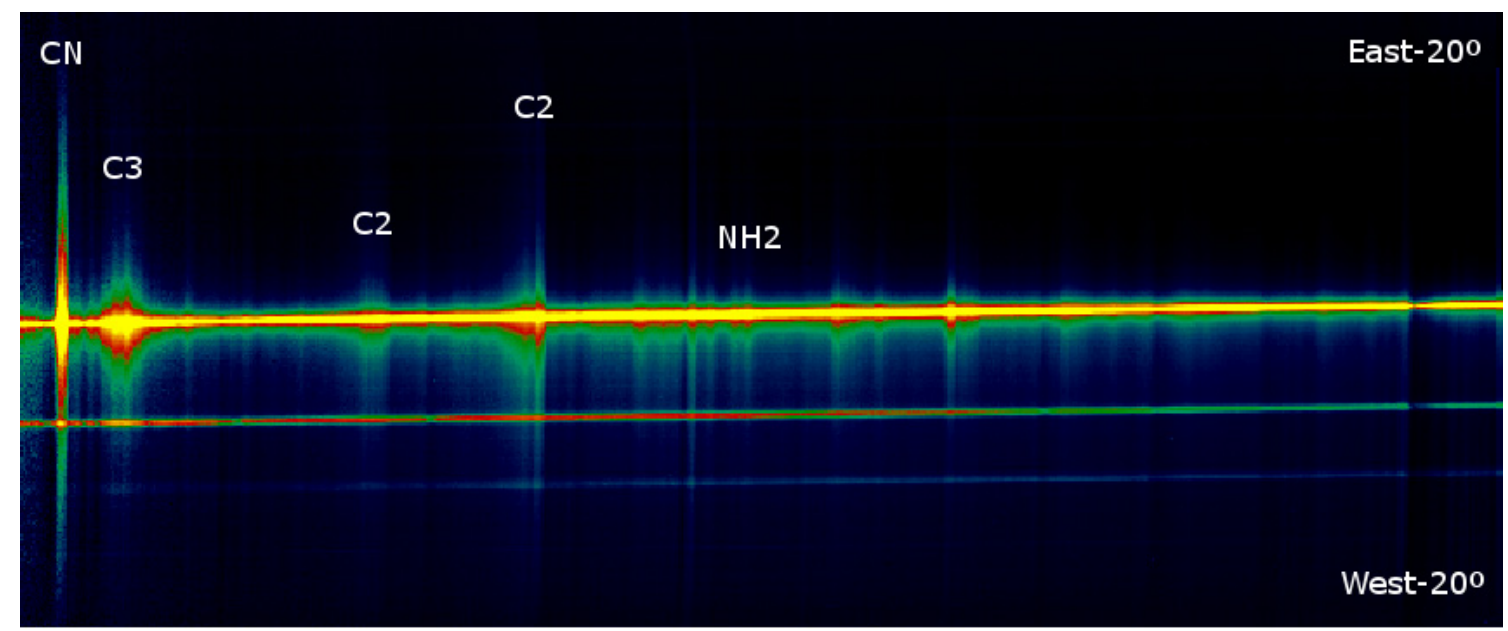

Fig. 9. Flux-calibrated spectrum of comet 103P/Hartley 2 acquired on October 27, 2010 where the strongest cometary emissions in the optical range $\left(\mathrm{CN}, \mathrm{C}_{2}, \mathrm{C}_{3}\right.$, and $\left.\mathrm{NH}_{2}\right)$ are marked. The slit was positioned as indicated in Fig. 1. The spatial scale covers from the optocentre up to $\sim 12000 \mathrm{~km}$ in both directions. The spatial resolution is $\sim 23 \mathrm{~km} /$ pix. The spectral wavelength range runs from 366.0 to $780.3 \mathrm{~nm}$ with resolution $0.33 \mathrm{~nm} /$ pix. The colour code stretches from 0 to $5 \times 10^{-16} \mathrm{erg} \mathrm{cm}^{-2} \mathrm{~s}^{-1} \AA^{-1}$.

long scale lengths, $\mathrm{CN}, \mathrm{C}_{2}$, and $\mathrm{NH}_{2}$, the theoretical profiles predict column densities which fall with $\rho$ in a much less pronounced way than the observed ones. However, better fits are obtained for $\mathrm{C}_{3}$ at every $\rho$ as the parent and daughter scale lengths are shorter than for the other analysed species.

On October 27, the fit is less accurate for $\mathrm{CN}$ than on October 29 as the enhanced emission of the two cones structures falls within the slit given its orientation in the PA 70-250 . This does not happen on October 29 when the slit is placed exactly in the E-W direction, that is, perpendicular to the direction where $\mathrm{CN}$ structures are detected. Furthermore, regarding $\mathrm{C}_{3}$, the situation is reversed, and the disagreement between Haser modelling and observations is greater on October 29 than on October 27, 2010.

Based on the results in A'Hearn et al. (1995) and in this work (see Table 4), we can conclude that, for a rather similar $\mathrm{OH}$ production rates in both passages $\left(2.1 \times 10^{28} \mathrm{~s}^{-1}\right.$ in 1991 and $1.8 \times 10^{28} \mathrm{~s}^{-1}$ in 2010), the production rates $Q$ of $\mathrm{CN}, \mathrm{C}_{3}, \mathrm{C}_{2}$, and $\mathrm{NH}_{2}$ measured at the 2010 perihelion passage are slightly higher than the ones measured during the 1991 perihelion passage.
Table 4. Comparison of gas and dust activity of 103P/Hartley 2 at the 1991 (A'Hearn et al. 1995) and 2010 perihelion passages.

\begin{tabular}{|c|c|c|c|c|c|c|}
\hline Date & & $\log _{1}$ & roduction & rates & & $\log Q(\mathrm{OH})$ \\
\hline & $\mathrm{CN} / \mathrm{OH}$ & $\mathrm{C}_{2} / \mathrm{OH}$ & $\mathrm{C}_{3} / \mathrm{OH}$ & $A f \rho / C N$ & $\mathrm{C}_{2} / \mathrm{CN}$ & \\
\hline 1991 & -2.45 & -2.36 & -3.56 & -23.47 & 0.08 & 28.31 \\
\hline 2010 & -3.24 & -3.11 & -4.21 & -22.88 & 0.12 & $28.25^{a}$ \\
\hline
\end{tabular}

Notes. ${ }^{(a)}$ From Biver et al. (2010, priv. comm.).

Mumma et al. (2011) report spectroscopic observations of HCN on UT 22.5 October. The HCN production rate on this preperihelion date is $(1.7 \pm 0.1) \times 10^{25} \mathrm{~s}^{-1}$. They oriented the slit along the sun-comet line, and the retrieved profiles were rather symmetric around the nucleus. The $Q_{\mathrm{CN}}$ obtained in this work is consistent with the Mumma et al. (2011) results that assume that $\mathrm{HCN}$ is the main parent species of $\mathrm{CN}$ and that the expected variations of $Q_{\mathrm{HCN}}$ around the perihelion are not very large. Our $\mathrm{CN}$ spectroscopic measurements also agree with the fact that 

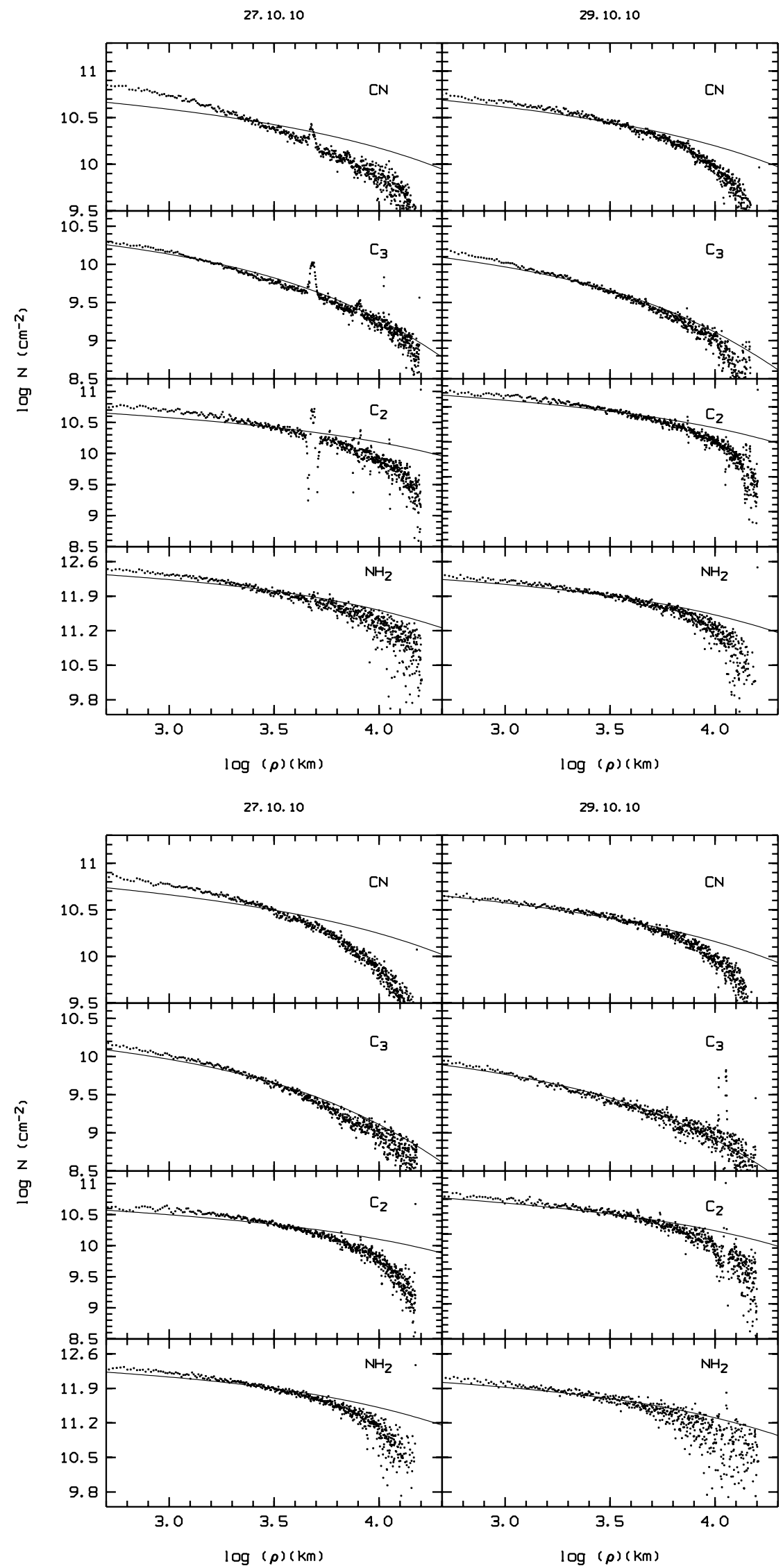

Fig. 10. Observed gas column density profiles $N$ in units of $\mathrm{cm}^{-2}$ versus the projected cometocentric distance $\rho$ in log-log representation in $70^{\circ}$ and in east direction for October 27 and 29, 2010, respectively. The best achievable fit obtained by means of the Haser modelling (Haser 1957) with parameters given in the text and production rates listed in Table 3 is plotted with solid lines.
Fig. 11. Observed gas column density profiles $N$ in units of $\mathrm{cm}^{-2}$ versus the projected cometocentric distance $\rho$ in $\log$ - log representation in $250^{\circ}$ and in west direction for October 27 and 29, 2010, respectively. The best achievable fit (solid line) obtained by means of the Haser modelling (Haser 1957) with parameters given in the text and production rates listed in Table 3. the $\mathrm{CN}$ (assuming $\mathrm{HCN}$ as the parent species) asymmetry lies in the north-south direction and not in sun-tail one. It has been reported that the $\mathrm{CN}$ jet appearance changes in morphology as a function of time with a periodicity of $\sim 17.6 \mathrm{~h}$ (Samarasinha et al. 2011) around the perihelion date, thus the north-south jet of CN on UT 27.21 October would have been placed at the 
same orientation on UT 22.81 October, about five hours later than the Mumma et al. (2011) observations. Biver et al. (2010, priv. comm.) have indicated that $\mathrm{HCN}$ production rate varied in the range $(1-2) \times 10^{25} \mathrm{~s}^{-1}$ with a period of $17.6 \mathrm{~h}$. These variations peaked around UT 25.10 and 27.24 October (i.e. three nucleus rotations) and broad minima were found around UT 25.4, 26.2, and 26.9 October. The comet spectrum on October 27 was acquired at about the same time as the reported maximum on UT 27.24 October. Comparing the $\mathrm{CN}$ production in Table 3 and the maxima of $\mathrm{HCN}$ production rates, we can thus conclude that on UT 27.24 October the $50-100 \%$ of the HCN outgassed from the nucleus is producing $\mathrm{CN}$.

The quotients of the $\mathrm{C}_{2}$ and $\mathrm{CN}$ gas production rates slightly increases by $5 \%$ from October 27 to 29 , which we cannot consider as representative of comet outgassing variations because of the error in the flux calibration and $Q_{i}$ determination (5-7\%) and because of the varying weather conditions during the $20 \mathrm{~min}$ integration time for the spectrum acquisition. A mean value of $\mathrm{C}_{2} / \mathrm{CN} \sim 1.2$ is given in A'Hearn et al. (1995), and $\sim 1.3$ is reported by Meech et al. (2011); therefore our determination can be considered as realistic, aside from the unstable sky conditions on October 29. According to this quotient, $\mathrm{C}_{2} / \mathrm{CN}$, comet 103P/Hartley 2 can be classified as typical, in terms of abundance of long-chain hydrocarbons, following the taxonomic types identified in A'Hearn et al. (1995).

The $\log \left[Q\left(\mathrm{C}_{2}\right) / A f \rho\right]$, with $A f \rho$ measured in the $520-525 \mathrm{~nm}$ spectral range, provides us with an approximate indication of the gas or dust enrichment of comet 103P. The result of this quotient is 23. In line with A'Hearn et al. (1995), the gas-to-dust mass ratio can be evaluated by

$\log \left(\frac{M_{\text {gas }}}{M_{\text {dust }}}\right)=\log [Q(\mathrm{OH}) / A f \rho]-25.4$.

According to this expression and using $Q\left(\mathrm{H}_{2} \mathrm{O}\right)=(1-2) \times$ $10^{28} \mathrm{~s}^{-1}$ (Biver et al. 2010, priv. comm.) and $A f \rho$ from results in Table 3, we compute a gas-to-dust mass ratio of $\sim 3-6$, a relatively gas-rich comet.

\section{Summary}

The comet 103P/Hartley 2, target of the EPOXI mission (NASA) was observed for three days around its perihelion, from October 27 to 29, 2010. The images and long-slit spectra that we acquired have provided us with the following picture of the comet at a distance of 1.058 AU from the Sun.

Both the gas and dust coma show features. The $\mathrm{CN}$ and $\mathrm{C}_{3}$ coma is not spherically symmetric, but a double jet in the northsouth direction is detected for both species on October 27 and 29, 2010. The CN double jet (or cone) changes morphology from 27 to 29 October: on October 27, the over-abundance of $\mathrm{CN}$ is more pronounced at the southern hemisphere than at the northern one, whereas on October 29, the two cones show similar $\mathrm{CN}$ over-abundance. The $\mathrm{C}_{3}$ coma displays a north-south asymmetry similar to the $\mathrm{CN}$ coma on October 29 , since it was not being possible to determine its existence on October 27 because no $\mathrm{C}_{3}$ images were acquired. The dust coma shows the tail in the anti-sunward direction, and a short Sunward jet curved to the south, which turns on and off following the comet nucleus periodicity (i.e. about $\sim 18.3 \mathrm{~h}$ at the time of the perihelion).

The Afo parameter, a proxy to the dust production rate, measured in a circular aperture of $\sim 4700 \mathrm{~km}$ ranges from $\sim 60 \mathrm{~cm}$ in the blue and $\sim 110 \mathrm{~cm}$ in the red. However, $A f \rho$ is rather stable in the innermost coma (equivalent aperture radius of $\sim 400 \mathrm{~km}$ ) when it is computed from the spectroscopic measurements within several continuum spectral ranges from 482 to $685 \mathrm{~nm}$. The strong variation in the Afo parameter between the blue and red spectral ranges when considering large aperture radius indicate a considerably dust reddening of $\sim 24 \% / 100 \mathrm{~nm}$. This normalized dust reflectivity varies with $\rho$ in the Sunward and tailward directions. Both directions display a blueing of the cometary dust, more noticeably in the Sun's direction.

The BC and RC surface brightness profiles, computed from azimuthally averaged profiles of the comet images, show a nominal behaviour of $\log B \sim-1.0 \log \rho$. However, a detailed study in sunward and tailward direction clearly shows that this slope $m$ is flatter $(m \sim-0.5$ to -0.6$)$ along the tail and steeper $(m \sim-1.1$ to -1.3$)$ towards the Sun.

The gas $\left(\mathrm{CN}, \mathrm{C}_{3}, \mathrm{C}_{2}\right.$, and $\left.\mathrm{NH}_{2}\right)$ production rates measured at perihelion, $r_{\mathrm{h}}=1.058 \mathrm{AU}$, are a bit higher than those during the 1991 perihelion passage (A'Hearn et al. 1995). The quotient $Q_{\mathrm{C}_{2}} / Q_{\mathrm{CN}} \sim 1.3$ places $103 \mathrm{P} /$ Hartley 2 as a typical comet in terms of long-chain hydrocarbons abundance. The gas-to-dust mass ratio is $\sim 3-6$, allowing us to consider $103 \mathrm{P}$ as a gas-rich comet.

Acknowledgements. This research has been supported by the Spanish Ministerio de Ciencia e Innovación under contract AyA 2009-08011. Z.-Y. Lin acknowledges a grant by the Junta de Andalucia under the project JA2007-TIC2744. K. J. Meech acknowledges support from NASA's Discovery Program through contract NNM07AA99C. L. M. Lara is very grateful to the WHT staff, especially to O. Vaduvescu, S. Rix, and F. Riddick for their support before and during the observations. L. M. Lara also acknowledges J. M. Bacaicoa for his help in editing the images.

\section{References}

A'Hearn, M. F. 2007, Icarus, 187, 1

A'Hearn, M. F., Schleicher, D. G., Millis, R. L., Feldman, P. D., \& Thompson, D. T. 1984, AJ, 89, 579

A'Hearn, M. F., Hoban, S., Birch, P. V., et al. 1986, Nature, 324, 649

A'Hearn, M. F., Millis, R. L., Schleicher, D. G., Osip, D. J., \& Birch, P. V. 1995, Icarus, 118, 223

A'Hearn, M. F., Belton, M. J. S., Delamere, W. A., et al. 2005, Science, 310, 258 Bertini, I., Lara, L. M., Vincent, J., et al. 2009, A\&A, 496, 235

Carusi, A., Kresak, L., Perozzi, E., \& Valsecchi, G. B. 1985, Long-term evolution of short-period comets, ed. A. Carusi, L. Kresak, E. Perozzi, \& G. B. Valsecchi

Colangeli, L., Epifani, E., Brucato, J. R., et al. 1999, A\&A, 343, L87

Crovisier, J., Brooke, T. Y., Leech, K., et al. 2000, Thermal Emission Spectroscopy and Analysis of Dust, Disks, and Regoliths, 196, 109

Festou, M. C. 1981a, A\&A, 95, 69

Festou, M. C. 1981b, A\&A, 96, 52

Groussin, O., Lamy, P., Jorda, L., \& Toth, I. 2004, A\&A, 419, 375

Harmon, J. K., Nolan, M. C., Howell, E. S., Giorgini, J. D., \& Taylor, P. A. 2011, ApJ, 734, L2

Hartley, M. 1984, IAU Circ., 4015, 1

Haser, L. 1957, Bull. Soc. Roy. Sci. Liège, 43, 740

Korsun, P. P., \& Jockers, K. 2002, A\&A, 381, 703

Kurucz, R. L., Furenlid, I., Brault, J., \& Testerman, L. 1984, Solar flux atlas from 296 to $1300 \mathrm{~nm}$, National Solar Observatory Atlas, Sunspot, New Mexico: National Solar Obsvervatory

Lara, L. M., Schulz, R., Stüwe, J. A., \& Tozzi, G. P. 2001, Icarus, 150, 124

Lara, L. M., Lin, Z., Rodrigo, R., \& Ip, W. 2011, A\&A, 525, A36

Larson, S. M., \& Sekanina, Z. 1984, AJ, 89, 571

Lin, Z. Y., Weiler, M., Rauer, H., \& Ip, W. H. 2007, A\&A, 469, 771

Lin, Z., Lin, C., Ip, W., \& Lara, L. M. 2009, AJ, 138, 625

Lisse, C. M., Fernandez, Y. R., Reach, W. T., et al. 2009, PASP, 121, 968

Meech, K. J., AHearn, M. F., Adams, J. A., et al. 2011, ApJ, 734, L1

Mumma, M. J., Bonev, B. P., Villanueva, G. L., et al. 2011, ApJ, 734, L7

Roettger, E. E. 1991, Ph.D Thesis (Baltimore, MD: Johns Hopkins Univ.)

Samarasinha, N. H., Mueller, B. E. A., A’Hearn, M. F., Farnham, T. L., \& Gersch, A. 2011, ApJ, 734, L3

Schleicher, D. G. 1983, Ph.D. Thesis, AA (Maryland Univ., College Park)

Snodgrass, C., Meech, K., \& Hainaut, O. 2010, A\&A, 516, L9

Thomas, P. C., Veverka, J., Belton, M. J. S., et al. 2007, Icarus, 187, 4

Turner, N. J., \& Smith, G. H. 1999, AJ, 118, 3039 